\title{
Neutron Protein Crystallography
}

\section{Hydrogen, Protons, and Hydration in Bio-macromolecules}

\author{
NOBUO NIIMURA \\ Frontier Research Center for Applied Atomic Sciences, \\ Ibaraki University, Japan \\ ALBERTO PODJARNY \\ Institut de Génétique et de Biologie Moléculaire et Cellulaire (IGBMC), \\ Institut National de Santé et de Recherche Médicale (INSERM) U964/ \\ Centre National de Recherche Scientifique (CNRS) UMR 1704/Université \\ de Strasbourg, 67404 Illkirch, France.
}




\section{Contents}

Part I: The basic principles and experimental configurations of neutron diffraction experiments

I-1. Introduction to the book

I-2. A brief review of the physics of neutrons and neutron diffraction

I-2.1. Basic properties of neutrons

I-2.1.1. Mass

I-2.1.2. Charge

I-2.1.3. Spin

I-2.1.4. Magnetic moment

I-2.2. Neutron-scattering length

I-2.3. Neutron absorption

I-2.4. Summary of the comparison of neutron and X-ray diffraction phenomena

I-2.5. Summary of the specific features of the interactions between neutrons and bio-macromolecules

I-2.6. Prerequisite concepts for understanding this book

I-2.7. The basic principles for analysis of the structure of single crystals

I-3. The production of neutrons

I-3.1. Steady-state reactor neutron sources: fission from nuclear criticality

I-3.2. Pulsed, accelerator-driven neutron sources: spallation from energetic protons

I-4. The moderation of neutrons

I-5. The transport of neutrons

I-5.1. Neutron beam tubes

I-5.2. Neutron bandpass filters (for reactor sources)

I-5.2.1. Narrow-bandpass wavelength filters (monochromators)

I-5.2.2. Wide-bandpass wavelength filters and the quasi-Laue method

I-5.3. Neutron choppers and the time-of-flight method (for accelerator-driven spallation sources)

I-5.3.1. The time-of-flight (TOF) method

I-5.3.2. The time analyzer

I-5.3.3. The TOF single-crystal diffractometer 
I-5.3.4. Summary: characteristics of the

TOF method

I-6. The detection of neutrons

I-6.1. The physics of detecting neutrons 32

I-6.2. Types of detectors $\quad 35$

I-6.2.1. Gas-proportional detectors 36

I-6.2.1.1. The resistive-wire type of PSD, based on the gas-proportional detector

I-6.2.1.2. The multi-anodes type of PSD, based on the gas-proportional detector

I-6.2.2. Scintillator detectors

I-6.2.2.1. The encode type of PSD, based on the scintillator detector

I-6.2.2.2. The wavelength-shift-fiber type of PSD, based on the scintillator detector

I-6.2.2.3. The Anger-camera type of PSD, based on the scintillator detector

I-6.2.3. Image-plate detectors

I-6.2.4. Comparison of the types of detectors

I-7. Summary comparison of the use of reactor sources and pulsed sources for neutron diffraction experiments

Part II: Experimental procedure for neutron protein crystallography

II-1. Flow chart of the experimental procedure for neutron protein crystallography

II-2. Crystallization

II-2.1. Crystallization phase diagram (CPD)

II-2.2. How to determine a CPD

II-2.2.1. Dialysis method

II-2.2.2. Vapor-diffusion method

II-2.2.3. Determination of a solubility curve (SC)

II-2.2.4. A special device for determination of a CPD

II-2.3. Two examples of CPDs

II-2.4. A comment about crystallization on the basis of a CPD

II-2.5. How to grow a large single crystal 60

II-2.5.1. Dialysis method 
II-3. Assessment of the quality of crystals

II-3.1. Introduction

II-3.2. Choosing a proper metric for judging the quality of crystals

II-3.3. Two examples of using the B-factor from the Wilson plot to judge crystal quality

II-3.3.1. B-DNA decamer d(CCATTAATGG) 69

II-3.3.2. Hen egg-white lysozyme (HEWL) 73

II-4. New techniques for sample preparation

II-4.1. Deuteration

II-4.2. Neutron crystallography at cryogenic temperatures $(\sim 15 \mathrm{~K})$

II-4.3. High-pressure cooling of protein crystals without cryo-protectant

II-5. Diffractometers

II-5.1. Comments about diffractometers in general

II-5.2. The BIX type of diffractometer

II-5.3. The quasi-Laue type of diffractometer 81

II-5.4. The TOF type of diffractometer

II-5.4.1. The Protein Crystallographic Station (PCS) diffractometer at the LANSCE pulsed neutron source

II-5.4.2. The Ibaraki Biological Crystal Diffractometer (iBIX) at J-PARC

II-5.4.3. The Macromolecular Neutron Diffractometer (MaNDi) at SNS

II-5.5. A comparison of existing diffractometers

$\begin{array}{ll}\text { II-6. Conducting the diffraction experiment } & 90 \\ \text { II-6.1. Mounting and aligning a crystal } & 90\end{array}$

II-6.2. Estimating the minimum required irradiation time $\quad 92$

II-7. Neutron data processing $\quad 93$

II-7.1. General view 93

II-7.2. Integrated intensity of Bragg reflections 93

II-7.2.1. Background subtraction 94

II-7.2.2. Lorentz-factor correction 97

II-7.2.3. $i(\lambda)$ and detector-efficiency corrections $\quad 97$

II-7.2.4. Absorption and extinction corrections $\quad 97$

II-7.3. Completeness and resolution 98

II-7.4. Example 1: RNase A, analyzed with the BIX diffractometer 99

II-7.4.1. Data collection 99

II-7.4.2. Data processing 99

II-7.5. Example 2: Antifreeze protein, analyzed with the LADI diffractometer 
II-7.5.1. Crystal data 101

II-7.5.2. Data collection 101

II-7.5.3. Data processing 102

$\begin{array}{ll}\text { II-8. Structure determination } & 103\end{array}$

II-8.1. The initial model 103

II-8.2. The analysis program 104

II-8.3. The determination of mobile hydrogen atoms 105

II-8.3.1. Hydrogen atoms in methyl groups $\quad 106$

II-8.3.2. Amide hydrogen atoms in the backbone 108

II-8.4. The determination of protonation states 108

II-8.5. The determination of hydrogen (deuterium) atoms in water molecules $\quad 109$

II-8.6. Improvements in the visibility of hydrogen atoms 112

II-8.7. The determination of the H/D exchange ratios 115

II-8.8. XN joint refinement 116

Part III: Hydrogen, protons, and hydration in bio-macromolecules 124

$\begin{array}{ll}\text { III-1. History of NPC } & 124\end{array}$

III-2. Observation of hydrogen atoms in macromolecules 125

III-3. Hydrogen and Hydration Protein Data Base (HHDB) 127

III-3.1. General view 127

III-3.2. Classification of the visibility of hydrogen atoms $\quad 129$

III-3.3. Methyl $\mathrm{CH}_{3}$ group configuration 129

III-4. Hydrogen bonds 133

III-4.1. General comments 133

III-4.2. The potential number of H-bonds in amino acid residues 134

III-4.3. Bifurcated hydrogen bonds 136

III-4.4. Noncolinear hydrogen bonds 139

III-4.5. Frustrated hydrogen bonds 140

III-4.6. Low-barrier hydrogen bonds 140

III-5. Examples of H/D exchange 142

III-5.1. Introduction $\quad 142$

III-5.2. Rubredoxin 143

III-5.3. Correlation of H/D exchange ratios with NMR results 144

III-5.4. Unusual examples of H/D exchange 146

III-5.4.1. Histidine 146

III-5.4.2. Guanine 147

III-5.5. Time evolution of H/D exchange 147

III-5.5.1. RNase A 147

III-5.5.2. $\mathrm{T}_{6}$ porcine insulin $\quad 150$

III-6. Protonation states of certain amino acid residues 152

III-6.1. General view 152 
III-6.2. Protonation state of the $\mathrm{N}^{\delta}$ and $\mathrm{N}^{\varepsilon}$ atoms of histidine: the case of insulin in heavy water

III-6.3. Carboxylate groups of acidic residues

III-7. Water molecules of hydration

III-7.1. How a hydration molecule is observed experimentally

III-7.2. Classification

III-7.3. Dynamic behavior and/or disordered states of hydrations

III-7.4. Oligomeric DNA duplexes

III-7.4.1. The B-DNA decamer

$$
\text { d(CCATTAATGG) }{ }_{2}
$$

III-7.4.2. The Z-DNA hexamer d(CGCGCG)

III-7.5. Water structure in concanavalin A (ConA)

III-8. Detailed examples of NPC

III-8.1.1. Structure of the active site

III-8.1.2. Protonation states of His 12 and His119

III-8.1.3. H-bonding networks around His48 and Gln 101

III-8.1.4. Hydration structure

III-8.1.5. Hydrogen bonds in $\alpha$-helices and $\beta$-sheets

III-8.2. Insulin

III-8.2.1. The contribution of NPC to solving the classical problems in insulin

III-8.2.2. The protonation/deprotonation of histidine

III-8.2.3. Charge balance of the zinc positive charges

$\begin{array}{ll}\text { III-9. Other examples of proteins solved by NPC } & 174 \\ \text { III-9.1. Lysozyme } & 174\end{array}$

III-9.2. Xylose isomerase $\quad 175$

III-9.3. Aldose reductase 176

III-9.4. Cytochrome P450cam 176

III-9.5. Amicyanin 177

III-9.6. Dihydrofolate reductase (DHFR) 177

III-9.7. Protocatechuate 3,4-dioxygenase (3,4-PCD) 178

III-9.8. Human deoxyhemoglobin 178

III-9.9. Diisopropyl fluorophosphatase 179

III-9.10. Photoactive yellow protein 181

III-9.11. Pancreatic elastase 181

III-9.12. HIV-1 protease 184 
III-9.13. D-antifreeze protein 186

III-9.14. Thermostable rubredoxin 186

$\begin{array}{ll}\text { III-10. Future prospects } & 187\end{array}$

III-10.1. Challenging structures (crystals with large unit cells) $\quad 187$

III-10.2. "Next generation" spallation neutron sources $\quad 188$

III-10.3. Pharmaceutical application (from a private communication by G. Klebe)

Appendix A: Neutron scattering length of elements 198

Appendix B: List of bio-macromolecules determined by NPC and deposited in the PDB before May 5, $2010 \quad 206$

Appendix C: Typical examples of nuclear density maps of amino acid residues

Appendix D: The next-generation technique for detecting hydrogen atoms with NPC 220

Appendix E: Books on neutron diffraction and scattering-further reading 222

Index 